\title{
Demethylation of HIN-1 reverses paclitaxel- resistance of ovarian clear cell carcinoma through the AKT-mTOR signaling pathway
}

\author{
Chih-Ming Ho ${ }^{1,2,3}$, Chi-Jung Huang ${ }^{4,10}$, Shih-Hung Huang ${ }^{5}$, Shwu-Fen Chang ${ }^{6^{*}}$ and Wen-Fang Cheng ${ }^{7,8,9^{*}}$
}

\begin{abstract}
Background: Methylation of HIN-1 is associated with poor outcomes in patients with ovarian clear cell carcinoma (OCCC), which is regarded to be an aggressive, chemo-resistant histological subtype. This study aimed to evaluate whether 5-aza-2-deoxycytidine (5-aza-2-dC) can reverse methylation of the HIN-1 gene to restore chemo-sensitivity of OCCC and the possible mechanism.
\end{abstract}

Methods: In vitro flow cytometric analysis and evaluation of caspase-3/7 activity of paclitaxel-sensitive and resistant OCCC cell lines were performed. Methylation status and expression changes of HIN-1 in the OCCC cell lines treated with 5-aza-2-dC were evaluated, and immunohistochemical staining of HIN-1 in OCCC tissues was performed. In vivo tumor growth with or without 5-aza-2-dC treatment was analyzed, and Western blotting of AKT-mTOR signaling-related molecules was performed.

Results: G2-M phase arrest was absent in paclitaxel-resistant OCCC cells after treatment with the cytotoxic drug. The caspase activities of the chemo-resistant OCCC cells were lower than those of the chemo-sensitive OCCC cells when treated with paclitaxel. Methylation of $\mathrm{HIN}-1$ was noted in paclitaxel-resistant OCCC cell lines and cancerous tissues. 5-aza-2-dC reversed the methylation of $\mathrm{HIN}-1$, re-activated the expression of $\mathrm{HIN}-1$, and then suppressed the in vivo tumor growth of paclitaxel-resistant OCCC cells. Immunoblotting revealed that phospho-AKT473 and phospho-mTOR were significantly increased in $\mathrm{HIN}-1$-methylated paclitaxel-resistant OCCC cell lines. However, the expressions of phospho-AKT at Ser473 and Thr308 and phospho-mTOR decreased in the OCCC cells with a high expression of HIN-1.

Conclusions: Demethylating agents can restore the HIN-1 expression in paclitaxel-resistant OCCC cells through the HIN-1-AKT-mTOR signaling pathway to inhibit tumor growth.

Keywords: Ovarian clear cell carcinoma, 5-aza-2-deoxycytidine, HIN-1, AKT/mTOR, Hypoxia-inducing factor

\section{Background}

Ovarian carcinoma is the fourth most common cause of cancer death among women in the United States [1]. Cytoreductive surgery followed by platinum-based chemotherapy is the standard initial treatment and has improved survival in patients with ovarian cancer [2]. Recently, ovarian clear cell carcinoma (OCCC) has become the second most common subtype in North America, and

\footnotetext{
* Correspondence: cmbsfc21@tmu.edu.tw; wenfangcheng@yahoo.com

${ }^{6}$ Graduate Institute of Medical Sciences, School of Medicine, Taipei Medical

University, Taipei, Taiwan

"Department of Obstetrics and Gynecology, National Taiwan, University

Hospital, Taipei, Taiwan

Full list of author information is available at the end of the article
}

the second leading cause of death from ovarian cancer [3]. The overall incidence of OCCC has been reported to be higher in Taiwan and Japan [4-6].

The combination of paclitaxel and platinum, recognized as the gold standard regimen for ovarian cancer [7], is used to treat patients with all subtypes of ovarian neoplasms including OCCC. Compared to ovarian serous carcinoma, OCCC is relatively resistant to platinum or taxane-based chemotherapy, and this chemo-resistance is associated with a lower response rate to chemotherapy and a poor prognosis $[5,6,8,9]$. For second-line or salvage treatment, the response rate for recurrent or refractory OCCC is far lower than that for other histological tumors, 
and even in patients with platinum-sensitive OCCC the response rate is lower than $10 \%$ [10]. Therefore, in order to improve the survival of patients with OCCC, the development of novel treatment strategies for both first-line and salvage treatment for recurrent disease is urgently needed. To achieve this goal, the identification of targets associated with chemo-resistance and elucidation of the molecular mechanisms of this process are urgently required.

Candidate DNA methylation drivers of acquired cisplatin resistance in ovarian cancer identified by methylome and expression profiling has been reported recently [11]. Data on potential key drivers of chemo-resistance in OCCC that are silenced by DNA methylation are limited, and further evaluation as to their potential as therapeutic targets for drug resistance is needed. Preclinical and clinical studies strongly support the use of combination regimens, and have shown that the hypomethylating agents azacitadine and decitabine can restore platinum sensitivity in chemoresistant ovarian cancer cell lines, xenografts, and patients with ovarian cancer [12-15]. However, the proof of concept of the therapeutic effect in parental or resistant OCCC in vitro and in vivo has not yet been established.

We hypothesized that there may be a subset of epigenetic changes causally associated with the acquisition of chemo-resistance in OCCC. In order to identify the epigenetically altered genes driving paclitaxel resistance in OCCC, we analyzed acquired DNA methylation changes in a human paclitaxel-resistant OCCC cell line, and expression changes associated with acquired resistance or following resensitization with demethylating agents. Although target therapies are currently used in many cancers, the molecular pathogenesis of chemo-resistance in OCCC is still unclear. We recently reported that methylation of HIN-1 promoter is a novel epigenetic biomarker associated with poor outcomes in patients with OCCC, and that the ectopic expression of the HIN-1 gene increases paclitaxel sensitivity partly through the Akt pathway [16]. Therefore, the aim of this study was to examine whether 5-aza-2-dC could reverse methylation of the HIN-1 gene and regulate the $\mathrm{AKT} / \mathrm{mTOR}$ signaling pathway, and then restore the chemo-response to paclitaxel in OCCC.

\section{Methods}

\section{Cell lines and cultures}

ES2 and TOV21G cell lines were obtained from the American Type Culture Collection. All cells were maintained in a humidified atmosphere containing $5 \% \mathrm{CO}_{2}$ at $37{ }^{\circ} \mathrm{C}$. ES-2 cells were grown in McCoy's 5A medium with 10 \% FBS, and TOV21G maintained in MCDB 105/medium 199 supplemented with $10 \%$ heat-inactivated fetal bovine serum.

\section{Establishment of chemo-resistant tumor cell lines}

Paclitaxel-resistant ES-2 and TOV21G tumor cell lines were developed by continuous exposure to paclitaxel.
Briefly, ES-2 and TOV21G cells were exposed to increasing concentrations of paclitaxel, with an initial concentration of $0.001 \mu \mathrm{M}$. When the tumor cells regained exponential growth after paclitaxel treatment, the concentration of paclitaxel was doubled until the concentration reached $0.2 \mu \mathrm{M}$. The resulting paclitaxel-resistant tumor cell lines were named ES2TR160 and TOV21GTR200. The ES2TR160 and TOV21GTR200 cells were passaged weekly and treated monthly with respective concentrations of paclitaxel to maintain their paclitaxel chemo-resistance.

\section{Generation of HIN-1 over-expressing ES2 and ES2TR160 cell lines}

ES2 and ES2TR160 tumor cells were transfected with the HIN-1 gene to generate HIN-1 over-expressing ES2 and ES2TR160 transfectants as described previously [16].

\section{Reagents and antibodies}

ECL Western blotting detection reagents were purchased from Perkin Elmer (Boston, MA). Antibodies recognizing HIN-1, mTOR, phospho-mTOR (Ser2448), AKT, phosphoAKT (Ser473) (Thr308), and GAPDH were purchased from Cell Signaling Technology (Beverly, MA). A Cell Titer 96well proliferation assay kit was obtained from Promega (Madison, WI), and paclitaxel was obtained from Genetaxyl Cream Less Company.

\section{MTT assays for cytotoxicity and proliferation assays}

The sensitivities of various tumor cell lines to paclitaxel were first assessed by MTT assay. Briefly, cells (4000 cells/well) in a 96-well plate were exposed to paclitaxel at the indicated concentrations for $72 \mathrm{~h}$ at $37{ }^{\circ} \mathrm{C}$. The cells exposed only to the culture medium served as controls. MTT at a final concentration of $0.5 \mathrm{mg} / \mathrm{ml}$ was added to the cells and incubated at $37{ }^{\circ} \mathrm{C}$ for $3 \mathrm{~h}$. At the end of incubation, the cultured medium was removed and $200 \mu \mathrm{l}$ of DMSO (Sigma) was added to dissolve the blue formazan crystals, and the optical density was measured at $490 \mathrm{~nm}$ using a universal microplate reader (Elx800, Bio-tek Instruments). IC50 values (the concentration that produced a $50 \%$ reduction in absorbance) were analyzed and recorded. MTT assays were then performed for the proliferation of tumor cells treated with 5-aza-2-dC as described earlier. Briefly, ES2 and ESTR160 cells $\left(1 \times 10^{4}\right.$ cells/well in a 6-well plate) with or without $10 \mu \mathrm{M} 5$-aza-2$\mathrm{dC}$ (in growth media for $1,3,7,10$ or 14 days with $5 \%$ $\mathrm{CO}_{2}$ at $\left.37^{\circ} \mathrm{C}\right) .5 \mathrm{mg} / \mathrm{ml}$ of MTT solution was then added to each well and incubated at $37{ }^{\circ} \mathrm{C}$ for $3 \mathrm{~h}$. The medium was then aspirated and replaced with solubilization solution (DMSO). The plates were read on a Micro Elisa reader (Anthos 2001) at $570 \mathrm{~nm}$. 
Cell cycle analysis of tumor cells treated with 5-aza-2-dC by flow cytometry analysis

To evaluate the influence of 5-aza-2-dC on the cell cycle of the tumor cells, the tumor cells were treated with or without $10 \mu \mathrm{M} 5$-aza-2-dC for 3 days, washed with PBS, and fixed with $70 \%$ ethanol overnight at $4{ }^{\circ} \mathrm{C}$. The cells were then stained with propidium iodide $(50 \mu \mathrm{g} / \mathrm{ml})$ and RNase A (100 $\mu \mathrm{g} / \mathrm{ml}$; Roth, Karlsruhe, Germany) for $20 \mathrm{~min}$ at $4{ }^{\circ} \mathrm{C}$. The percentages of cells in the $\mathrm{G}_{0} / \mathrm{G}_{1}$ phase, $S$ phase, and $G_{2} / M$ phase were determined using a FACScan flow cytometer and analyzed by Cell Quest software (Becton Dickinson, San Jose, CA).

\section{Caspase-3/7 activity of the tumor cells treated with paclitaxel and/or 5-aza-2-dC}

Caspase-3/7 activity of the tumor cells was determined quantitatively using a Caspase-Glo 3/7 assay kit (Promega) according to the manufacturer's instructions. Briefly, the tumor cell lines were seeded and treated with paclitaxel or 5-aza-2-dC. After $24 \mathrm{~h}$, the cells were lysed and luminogenic substrates specific for the caspase species were added. Light emission was measured in a luminometer (Berthold Technologies, Wildbad, Germany).

\section{Genomic DNA and RNA extraction}

Genomic DNA and RNA was extracted from the tumor cell lines using a QIAamp tissue kit (Qiagen, Valencia, CA) following the instructions of the manufacturer.

\section{Sodium bisulfite treatment, sequencing, and methylation- specific polymerase chain reaction analysis}

Genomic DNA of the tumor cells was isolated using a Genomic DNA kit (Geneaid Biotech, Bade City, Taiwan), converted with sodium bisulfite using a CpGenome DNA modification kit (Millipore, MA, USA), purified, and then amplified by a PCR with DNA polymerase (ThermoHotStart 2x Gold PCR Master mix; Applied Biosystems) and HIN-1-specific primers. The primer sequences for methylated HIN-1 were 5'-GAAGTTTCGTGGTTTTGTTCG-3' (forward) and 5'-AAAACCTAAAATCCACGATCGAC-3' (reverse), and the primer sets for unmethylated HIN-1 were 5'-TAAGAAGTTTTGTGGTTTTGTTTGG-3' (forward) and 5'-AAAAAACCTAAAATCCACAATCAAC-3' (reverse). Bisulfite-modified Sss I (New England Biolabs, MA)-treated normal lymphocyte DNA served as the methylated control, and bisulfite-treated normal lymphocyte DNA as the unmethylated control. PCR products were analyzed on $3 \%$ agarose gels. A methylation specificPCR in a final volume of $20 \mu \mathrm{l}$ was performed under the following conditions: $95^{\circ} \mathrm{C}$ for $10 \mathrm{~min}$, followed by $40 \mathrm{cy}$ cles at $95{ }^{\circ} \mathrm{C}$ for $30 \mathrm{~s}, 62{ }^{\circ} \mathrm{C}$ for $30 \mathrm{~s}$, and $72{ }^{\circ} \mathrm{C}$ for $40 \mathrm{~s}$, with a final extension at $72{ }^{\circ} \mathrm{C}$ for $10 \mathrm{~min}$ and holding at $4{ }^{\circ} \mathrm{C}$. The PCR products were purified and then directly sequenced using an Applied Biosystems ABI automated DNA sequencer.

\section{Quantitative real-time RT-PCR (QRT RT-PCR)}

QRT RT-PCR was used to measure the MDR1, NANOG, HIF-1 $\alpha$, HIF-2 $\alpha$, Snai2, TWIST1, and ABCG2 mRNA of the tumor cell lines. GAPDH was used as the internal control. The QRT RT-PCR was performed in an ABI Prism 7300 Sequence Detection System (Applied Biosystems) with a Taqman Gene Expression Assay (Hs00369360_g1) under the following conditions: $2 \mathrm{~min}$ at $50{ }^{\circ} \mathrm{C}, 10 \mathrm{~min}$ at $95{ }^{\circ} \mathrm{C}$, and a two-step cycle at $95^{\circ} \mathrm{C}$ for $15 \mathrm{~s}$ and $60{ }^{\circ} \mathrm{C}$ for 1 min for 40 cycles with an additional dissociation curve. The interpolated number $(\mathrm{Ct})$ of cycles to reach a fixed threshold above background noise was used to quantify amplification.

\section{5-aza-2-dC treatment and QRT RT-PCR of HIN-1 of the tumor cell lines}

The tumor cell lines were treated with or without 5-aza-2$\mathrm{dC} 10 \mu \mathrm{M}$, and renewed every $24 \mathrm{~h}$. QRT RT-PCR was used to measure the mRNA of HIN-1 as described earlier.

\section{Immunohistochemistry}

Formalin-fixed, paraffin-embedded specimens were sliced by a microtome at a thickness of 3-5 um and placed on coated slides. The tissue slides were then incubated with purified goat anti-human UGRP2 (S-15) polyclonal Ab (Santa Cruz Biotechnology) using a Thermo Scientific Autostainer 360 (Thermo Fisher Scientific Inc., CA). The immuno-reactive HIN-1 was scored semi-quantitatively, and the expression was scored according to the intensity as 0 or 1,2 or 3 indicating no or low, intermediate or strong immuno-reactivity, respectively. Tissues containing more than $10 \%$ neoplastic cells with a score of 2-3 intensity were considered to be positive. The percentages of each score in the neoplastic tissues were also recorded. If less than $10 \%$ of the neoplastic cells expressed HIN-1 the expression was defined as being weak, and if more than $10 \%$ of the neoplastic cells expressed HIN-1 the expression was defined as being strong. A pathologist not involved in the present study evaluated the immunostaining under blinded conditions.

\section{Western blot analysis}

Tumor cell lines were first treated with paclitaxel or 5aza-2-dC for $72 \mathrm{~h}$. The cells were then collected and lysed in PBS containing $1 \%$ Triton X-100 using an ultrasonic cell disruptor. The lysates were separated by SDS-PAGE $(12.5 \%)$ and transferred to a PVDF membrane. The membrane was blocked in blocking buffer (TBS containing $0.2 \%$ Tween 20 and $1 \%$ I-block (NEN)) and incubated with the polyclonal antibodies separately for $1 \mathrm{~h}$. A purified rabbit anti-human GAPDH polyclonal Ab (Santa 
Cruz Biotechnology, Inc.) was also used at the same time to normalize the signals generated from anti-HIN-1, AKT, AKT p-Akt (Ser473), pAKT (Thr308), mTOR, and pMTOR (Cell Signaling). After washing, alkaline phosphatase-conjugated anti-rabbit Ab (Vector Laboratories) was applied. The membrane was washed and the bound Abs was visualized by developing with NBT/BCIP as chromogens.

\section{In vivo animal experiments}

NOD/SCID (NOD.CB17 Prkdc scid/Jnarl) mice were obtained from the National Animal Center (Taipei, Taiwan) and maintained in accordance with institutional policies. All of the experiments were approved by the Institutional Animal Care and Use Committee of Cathay General Hospital. Five to 7 -week-old NOD/SCID mice $(n=4)$ were inoculated subcutaneously into the bilateral flank with $1 \times 10^{7}$ of tumor cells treated with or without $10 \mu \mathrm{M}$ 5 -aza-2-dC for 3 days before inoculation. Tumor growth was measured using calipers, and volumes were calculated based on the modified ellipsoid formula $(\mathrm{L} \times \mathrm{W} \times \mathrm{W} / 2)$ at the indicated time points. All of the experiments were carried out in duplicate.

\section{Statistical analysis}

The median inhibitory concentrations (IC50) of paclitaxel were calculated using Sigma Plot 8.0 software (SPSS, Inc., Chicago, IL). All numerical data were expressed as the mean \pm SD. Significance of the difference between two groups was determined with the Mann-Whitney $U$ test. A $p$ value less than 0.05 was considered to be statistically significant.

\section{Results}

Characteristics of paclitaxel-sensitive and paclitaxelresistant cell lines in $\mathrm{IC}_{50}$, concentration, cell proliferation and distribution of cell cycle

The IC50 concentrations of parental ES2 cells, TOV21G, and their paclitaxel-resistant clones ES2TR160 and TOV21GTR200 cells are shown in supplement Table 1. The relative resistant indices of ES2TR160 vs. ES2 and TOV21GTR200 vs. TVO21G were 9.36 and 228.3, respectively. The cell morphologies of the cell lines treated with paclitaxel are shown in Fig. 1a. Damaged morphology was noted in the ES2 cells but not in the ES2TR160 cells, including a decline in cell number, and rounded cells undergoing hydropic and vacuolated changes (Fig. 1a). Cell proliferation assays of ES2 and ES2TR160 cells treated with $160 \mathrm{nM}$ of paclitaxel showed that the cell proliferative activity of the ES2 cells was significantly inhibited by paclitaxel compared with the ES2TR160 cells (Fig. 1b).

The percentages of sub-G1, G1 and G2 phases among the parental ES2 and ES2TR160 cells treated with different
Table 1 Clinico-pathological characteristics and $\mathrm{HIN}-1$ expression of 42 OCCC patients

\begin{tabular}{llll}
\hline Parameter & $\begin{array}{l}\text { Low HIN-1 } \\
\text { expression }\end{array}$ & $\begin{array}{l}\text { High HIN-1 } \\
\text { expression }\end{array}$ & $p$ value \\
\hline Patient numbers & 18 & 24 & \\
Age & $49(32-66)$ & $55(32-66)$ & $0.393^{\mathrm{a}}$ \\
$\begin{array}{l}\text { [years, median } \\
\text { (range) }\end{array}$ & & & \\
$\begin{array}{l}\text { Disease stage } \\
\text { Early (I+II) }\end{array}$ & 12 & 4 & $0.067^{\mathrm{b}}$ \\
$\quad$ Advanced (IIII + IV) & 14 & 12 & \\
Tumor size (cm) & $12.8(6-21)$ & $12.5(3-23)$ & $0.662^{\mathrm{a}}$ \\
\hline
\end{tabular}

OCCC ovarian clear cell carcinoma

ane-way ANOVA

${ }^{\mathrm{b}}$ Chi-square test

concentrations of paclitaxel were further analyzed. There was no significant difference in the frequency of G1 (56.0 \pm $1.8 \%$ vs. $51.0 \pm 1.4 \%)$ or G2 (20.1 $\pm 0.9 \%$ vs. $22.0 \pm 1.3 \%)$ phase in between the ES2 and ES2TR160 cells before treatment with paclitaxel (Fig. 1c), and the results were similar between TOV21GTR200 and TOV21G cells (data not shown). The percentage of the G2 phase in the ES2 cells treated with $160 \mathrm{nM}$ paclitaxel was significantly higher than that in the ES2 cells without paclitaxel treatment $(78.40 \pm$ $3.35 \%$ vs. $20.10 \pm 0.88 \%, p=0.0001$, one-way ANOVA) (Fig. 1d). In contrast, the percentage of the G2 phase in the ES2TR160 cells treated with $160 \mathrm{nM}$ paclitaxel was not significantly different compared with that in the ES2TR160 cells without paclitaxel treatment (22.75 \pm $0.44 \%$ vs. $22.02 \pm 1.27 \%, p=0.41$, one-way ANOVA) (Fig. 1d). These results indicated that G2-M phase arrest was absent in the paclitaxel-resistant OCCC cells after being treated with a cytotoxic drug.

\section{Caspase activity in chemo-sensitive cells was higher than} in chemo-resistant cells when treated with paclitaxel

The caspase activity in the tumor cells treated with paclitaxel was then evaluated. As shown in Fig. 2a, the caspase-3/7 activity in the ES2 tumor cells was significantly higher than in the ESTR160 tumor cells when treated with paclitaxel $(p<0.001$, one-way ANOVA).

\section{Drug resistance-related genes in paclitaxel-resistant tumor cells were more highly expressed than in paclitaxel-sensitive tumor cells}

The expression levels of drug resistance-related genes were further evaluated by QRT RT-PCR. The expression levels of MDR1 (Fig. 2b), NANOG (Fig. 2c), HIF-1 $\alpha$ (Fig. 2c), HIF-2 $\alpha$ (Fig. 2c), Snai2 (Fig. 2c), TWIST1 (Fig. 2d), and ABCG2 (Fig. 2e) were significantly higher in the paclitaxelresistant cell lines ES2TR160 and TOV21GTR200 than in the paclitaxel-sensitive cell lines ES2 and TOV21G. These results indicated that genes related to drug transport, 


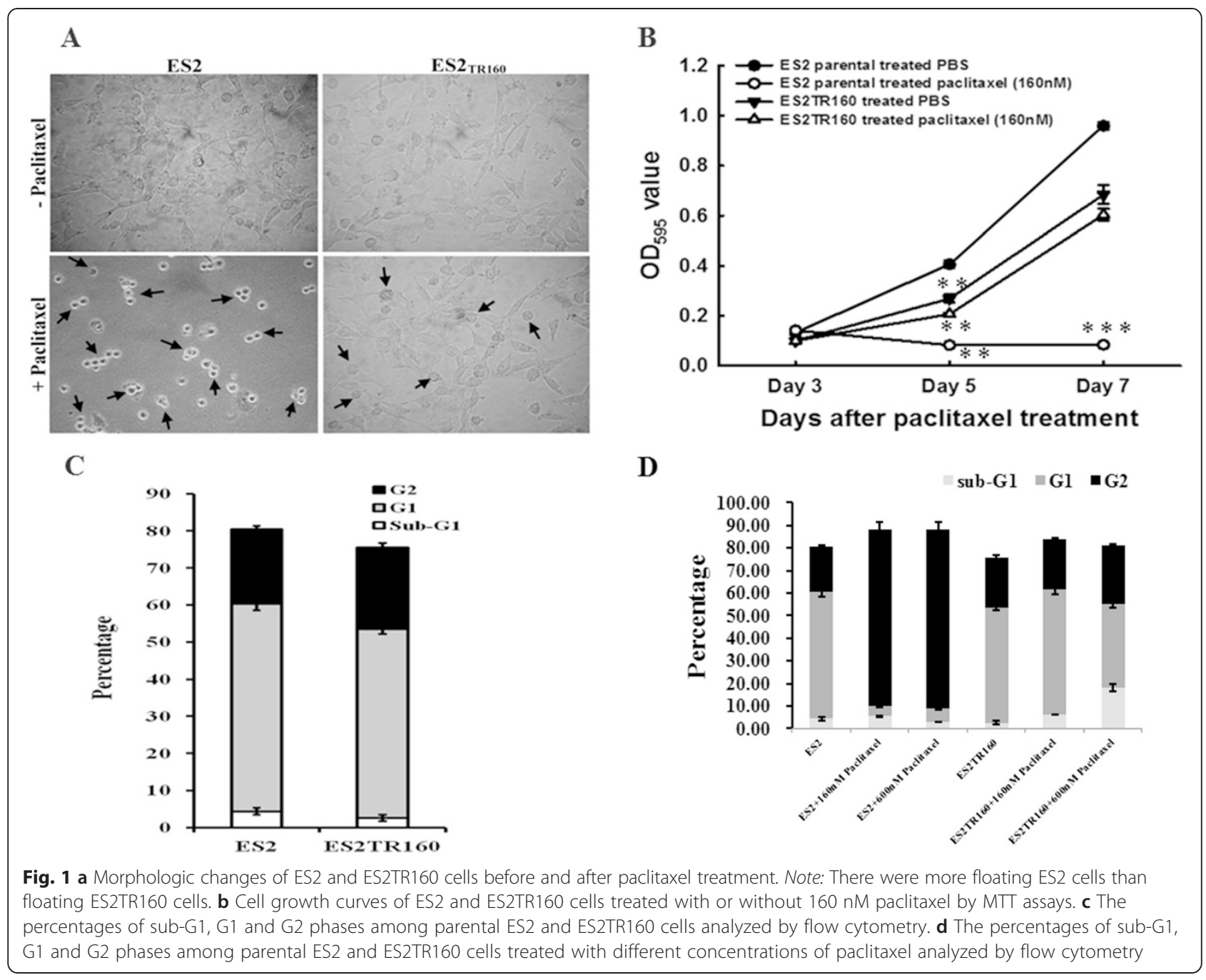

cancer stem cell characteristics, hypoxic tumor microenvironment, and epithelial-mesenchymal transition were highly expressed in the paclitaxel-resistant tumor cells.

\section{HIN-1 methylation of paclitaxel-resistant tumor cells could} be reversed by a demethylating agent

Changes in the methylation status of the HIN-1 gene in paclitaxel-sensitive and resistant tumor cell lines were evaluated by methylation-specific PCR. As shown in Fig. 3a, the ES2TR160 cells showed higher methylation of HIN-1 compared with the ES2 cells. We then tested whether a demethylating agent could reverse the methylation of the HIN-1 gene and then reactivate the expression of HIN-1. The methylation of HIN-1 in the ES2TR160 cells was reduced with 5-aza-2-dC treatment (Fig. 3a). In addition, the expression levels of HIN-1 in the 5-aza-2deoxycytidine-treated groups were significantly increased compared with those in the PBS-treated groups in both the ES2 and ES2TR160 cell lines (Fig. 3b and c). These results indicated that the expression of HIN-1 in paclitaxel- resistant OCCC cells was lower than that in paclitaxelsensitive OCCC cells due to the methylation of HIN-1. In addition, a demethylating agent could reverse the methylation of HIN-1 and restore its expression.

\section{Paclitaxel-resistant OCCC tissues expressed lower levels of} HIN-1 than paclitaxel-sensitive OCCC tissues

The representative photographs of HIN-1 immunoreactivity in OCCC tumor tissues by immunohistochemical staining are shown in Fig. 4 (Fig. 4a: high expression of HIN-1, Fig. 4b: low expression of HIN-1). Fourteen $(33.3 \%)$ of 42 patients were paclitaxel-resistant and 28 (66.7\%) were paclitaxel-sensitive. Among the 14 paclitaxelresistant OCCC tissues, 13 (93.8 \%) showed a weak HIN-1 protein expression. In contrast, among the 28 paclitaxelsensitive OCCC tissues, only $17(62.8 \%)$ showed a weak HIN-1 protein expression. The paclitaxel-resistant OCCC tissues had a significantly higher percentage of weak HIN-1 protein expression than the paclitaxel-sensitive OCCC tissues (93.8 \% vs. $62.8 \%, p=0.03$, chi-square test) (Fig. 4c). 

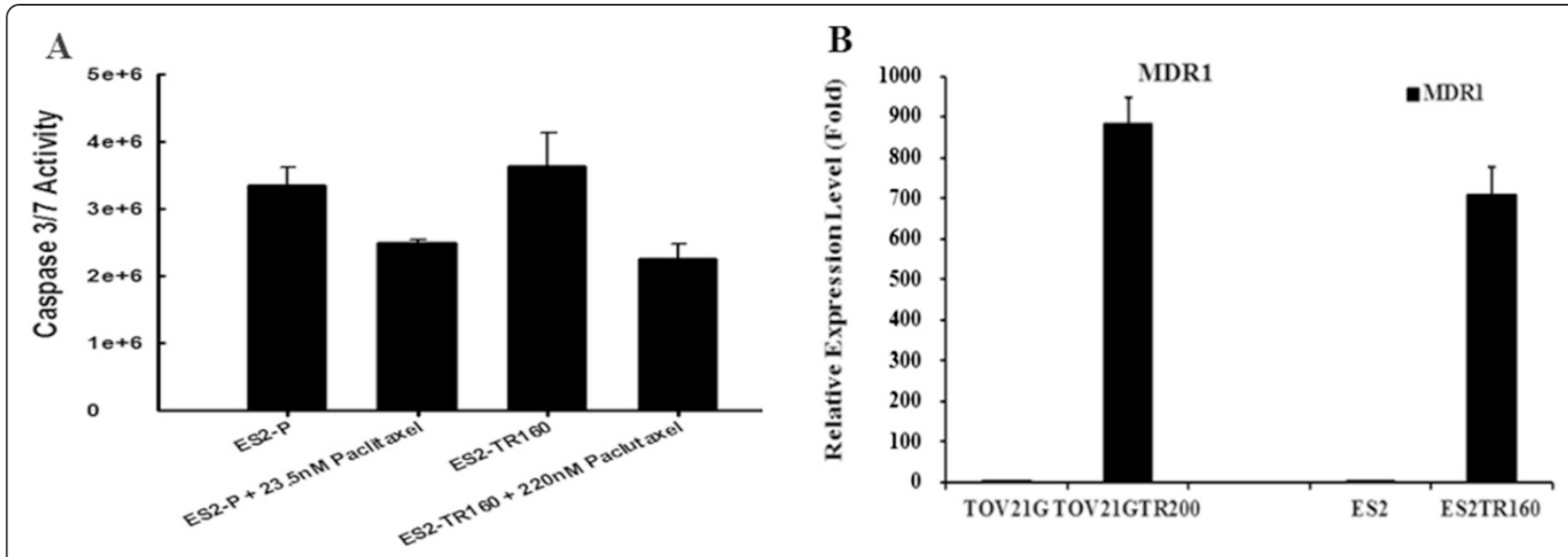

C

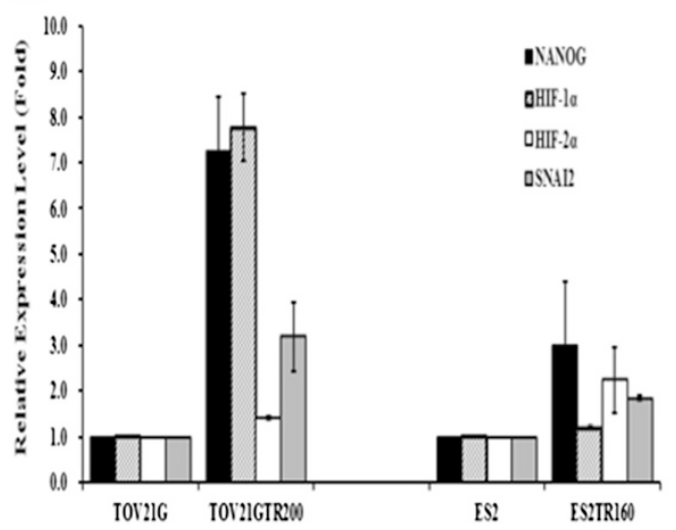

D

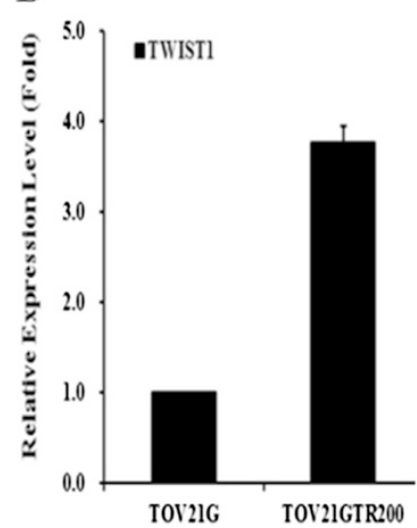

E

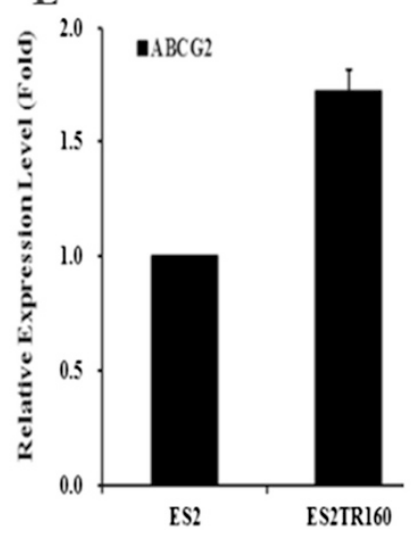

Fig. 2 a Caspase-3/7 activity in both ES2 and ESTR160 cells with or without paclitaxel treatment. The expression levels of (b) MDR1, (c) NANOG, HIF-1a, HIF-2a, and Snai2, (d) TWIST1, and (e) ABCG2 in ES2 and TOV21G parental cells and their derived paclitaxel-resistant ES2TR160 and TOV21GTR200 cells by QRT-PCR

These results indicated that the HIN-1 expression was strongly associated with the response to paclitaxel of the OCCC patients. We retrospectively reviewed and analyzed our 42 OCCC patients. Among the analyzed 26 advanced OCCC tissues, 14 (54\%) samples of advanced OCCCs showed scored as HIN-1 weak staining ( 0 or +1 immunoreactivity), and 12 (46\%) were $\mathrm{HIN}-1$ strong staining (+2 or +3 immuno-reactivity). In contrast, among the 16 early stage OCCCs, 12 (75\%) tumors were scored as 0 or +1 immuno-reactivity and $4(25 \%)$ were +2 or +3 . The percentage of HIN-1 immuno-reactivity at 0 or +1 was significantly higher in advanced OCCCs than in early stage (54\% vs $25 \%, \mathrm{p}=0.067)$. These results indicate that loss of HIN-1 expression has a trend towards advanced OCCC tumors. However, HIN-1 expression levels among tumors are not associated with tumor size $(p=0.662)$ (Table 1$)$.

\section{$\mathrm{HIN}-1$ reduced in vivo tumor growth}

To further examine whether HIN-1 could inhibit the growth of paclitaxel-resistant OCCC tumor cells, in vivo subcutaneous xenograft experiments were performed. Mice receiving ES2TR160 cells expressing high concentrations of HIN-1 had a smaller tumor size compared with those challenged with ES2TR160 parental cells (Fig. 5a) (ES2TR160 cells with high expressions of HIN-1 vs. ES2TR160 mock cells; day $21,133.76$ vs. $211.74 \mathrm{~mm}^{3}, p=0.036$; day 27 , 266.55 vs. $484.92 \mathrm{~mm}^{3}, p=0.008$, both by the Student's $t$ test). These results indicated that HIN-1 could inhibit the in vivo growth of paclitaxel-resistant OCCC tumor cells.

\section{5-Aza-2-dC inhibited the in vivo tumor growth of} paclitaxel-sensitive and resistant OCCC cell lines

The in vivo growth inhibitory effect of 5-aza-2-dC on OCCC tumor cells was further evaluated. As shown in Fig. 5b, the mice receiving ES2 or TOV21G parental tumor cells treated with 5-aza-2-dC had smaller tumor sizes compared with those treated with PBS. The mean tumor size of the ES2 tumor cells treated with 5-aza-2-dC was smaller than that of ES2 tumor cells treated with PBS (day $37,217.8$ vs. $1764.1 \mathrm{~mm}^{3}, p<0.01$, Student's $t$ test). In addition, the mice challenged with ES2TR tumor cells 
$\mathbf{A}$

\begin{tabular}{|c|c|c|c|c|c|}
\hline \multicolumn{3}{|c|}{ ES? } & \multicolumn{3}{|c|}{$\mathrm{ES}_{\mathrm{TR} 160}$} \\
\hline - & Day 3 & Day 6 & - & Day 3 & Day 6 \\
\hline M U & M U & M U & M U & M U & M U \\
\hline
\end{tabular}

B

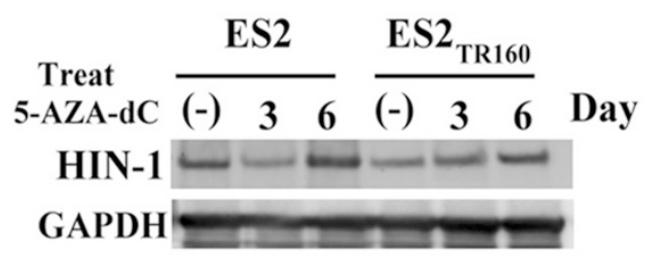

C

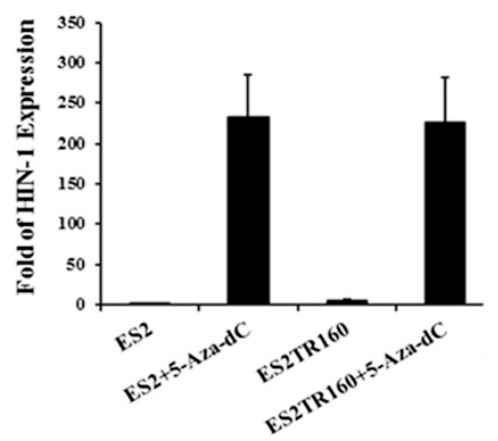

Fig. 3 a Changes of methylation status of the HIN-1 gene detected by methylation-specific PCR in parental ES2 cells and the derived paclitaxel-resistant ES2TR160 cells before and after 5-aza-2-dC treatment for 3 or 6 days. M represents methylation and $U$ represents unmethylation. b Representative figure of HIN-1 mRNA expression in ES2 and ES2TR160 cells treated with or without 5-aza-2-dC for 3 or 6 days. $\mathbf{c}$ Bar figure of the folds of HIN-1 mRNA expression in the ES2 and ES2TR160 cells treated with or without 5-aza-2-dC for 6 days
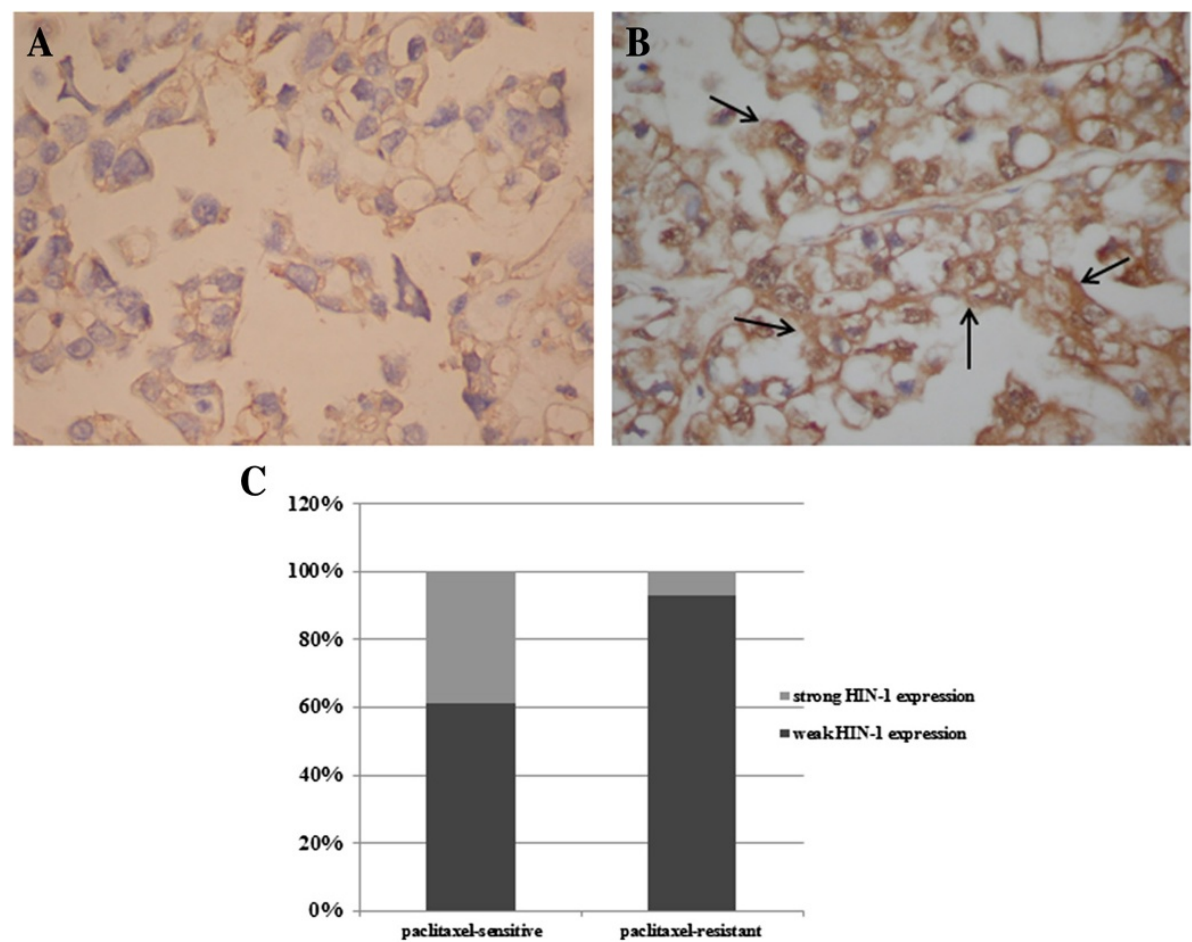

Fig. 4 Representative immunohistochemical staining of HIN-1 in OCCC cancerous tissues. a Weak expression of HIN-1. b High expression of HIN-1. Note: The high HIN-1 expression was noted in the cytoplasm of the neoplastic cells (arrows). c Bar figures of the percentage of weak and strong HIN-1 protein expression in paclitaxel-resistant and paclitaxel-sensitive OCCC tissues 


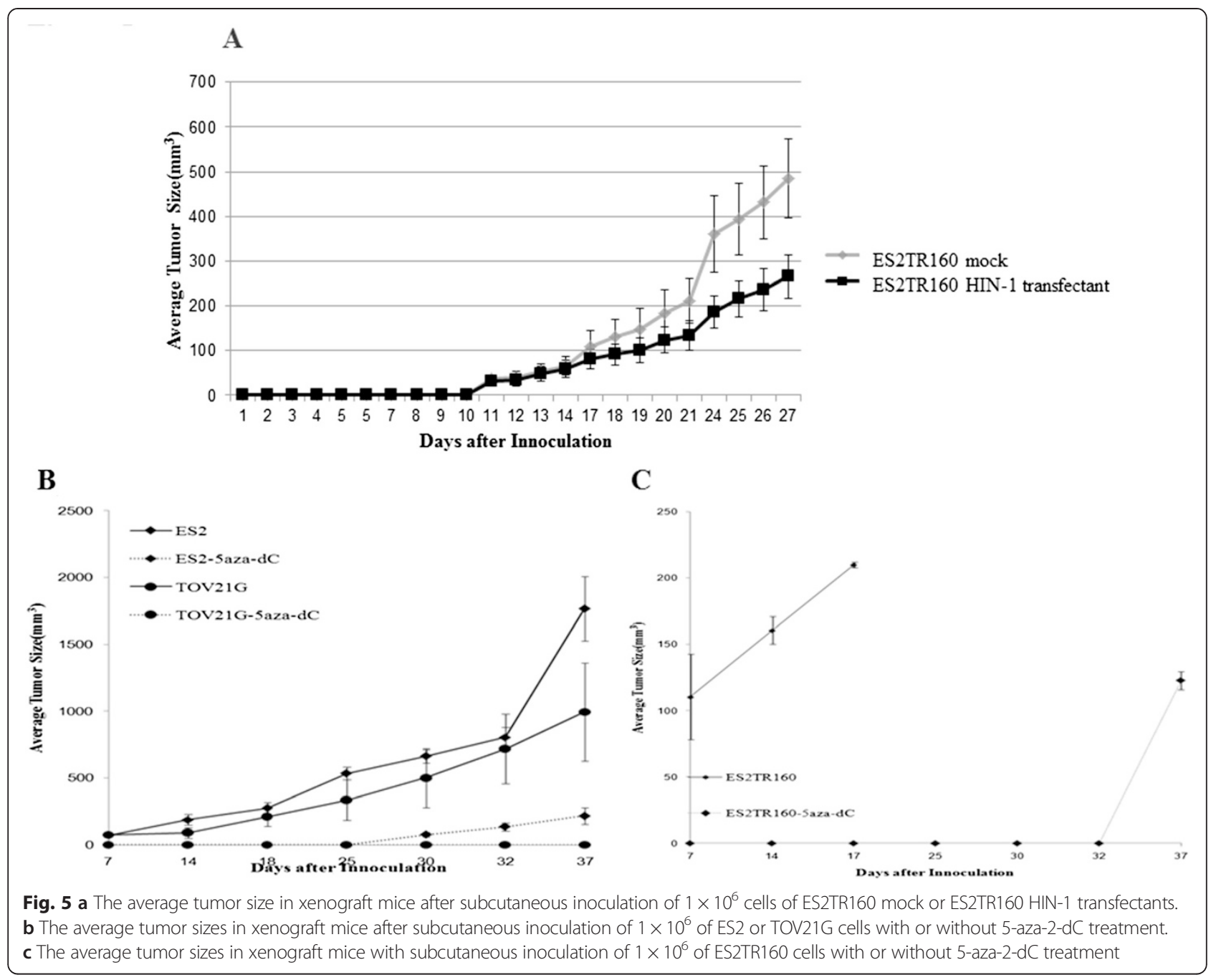

with PBS died on day 18. However, none of the mice challenged with ES2TR tumor cells treated with 5-aza-2-dC had died 35 days after tumor challenge (Fig. $5 \mathrm{c}$ ). These results suggest that 5 -aza-2-dC effectively inhibited the growth of both paclitaxel-sensitive and resistant OCCC tumor cells.

\section{5-Aza-2-dC inhibited the proliferative activities of both} paclitaxel-sensitive and resistant OCCC cell lines in vitro The effects of 5-aza-2-dC on the growth of paclitaxelsensitive and paclitaxel-resistant OCCC cells were examined by MTT assays. The results showed that 5 -aza-2-dC inhibited cell growth by $70 \%$ in both ES2 and ES2TR160 tumor cells (Fig. 6a). In addition, 5-aza-2-dC significantly reduced the percentages of the G1 phase $(66.4 \% \pm 1.1$ to $19.9 \% \pm 1.8 \%$ in the ES2 cells, $p<0.001 ; 50.8 \% \pm 2.7$ to $22.0 \% \pm 2.4 \%$ in the ES2TR160 cells, $p<0.001$, Student's $t$ test), but significantly increased the percentages of the G2 phase (14.4 \% \pm 0.5 to $39.0 \% \pm 1.7 \%$ in the ES2 cells, $p<$ $0.001 ; 17.4 \% \pm 0.6$ to $34.8 \% \pm 1.8 \%$ in the ES2TR160 cells, $p<0.01$, Student's $t$ test) after 3 days of treatment (Fig. 6b). Furthermore, 5-aza-2-dC also reduced the caspase-3/7 activity in ES2 cells but not in ES2TR160 cells (Fig. 6c).

\section{HIN-1-AKT-mTOR signaling pathway was involved in the paclitaxel-treated OCCC tumor cells}

The molecules involved in the signaling pathways associated with paclitaxel-related drug resistance were further evaluated by immunoblotting analysis. The expressions of phospho-AKT473 and phospho-mTOR were significantly increased in the ES2TR160 cells in parallel to the decrease in HIN-1 expression compared to the ES2 parental cells (Fig. 7a). However, the expressions of phospho-AKT at Ser473 and Thr308 and phosphomTOR were decreased in the ES-2TR160 tumor cells with high expressions of HIN-1 (Fig. 7a). In addition, 5-aza-2-dC also decreased the expressions of phosphoAKT at Ser473 and Thr308 and phospho-mTOR. Whereas, an increased HIN-1 expression was observed in the 5-aza- 

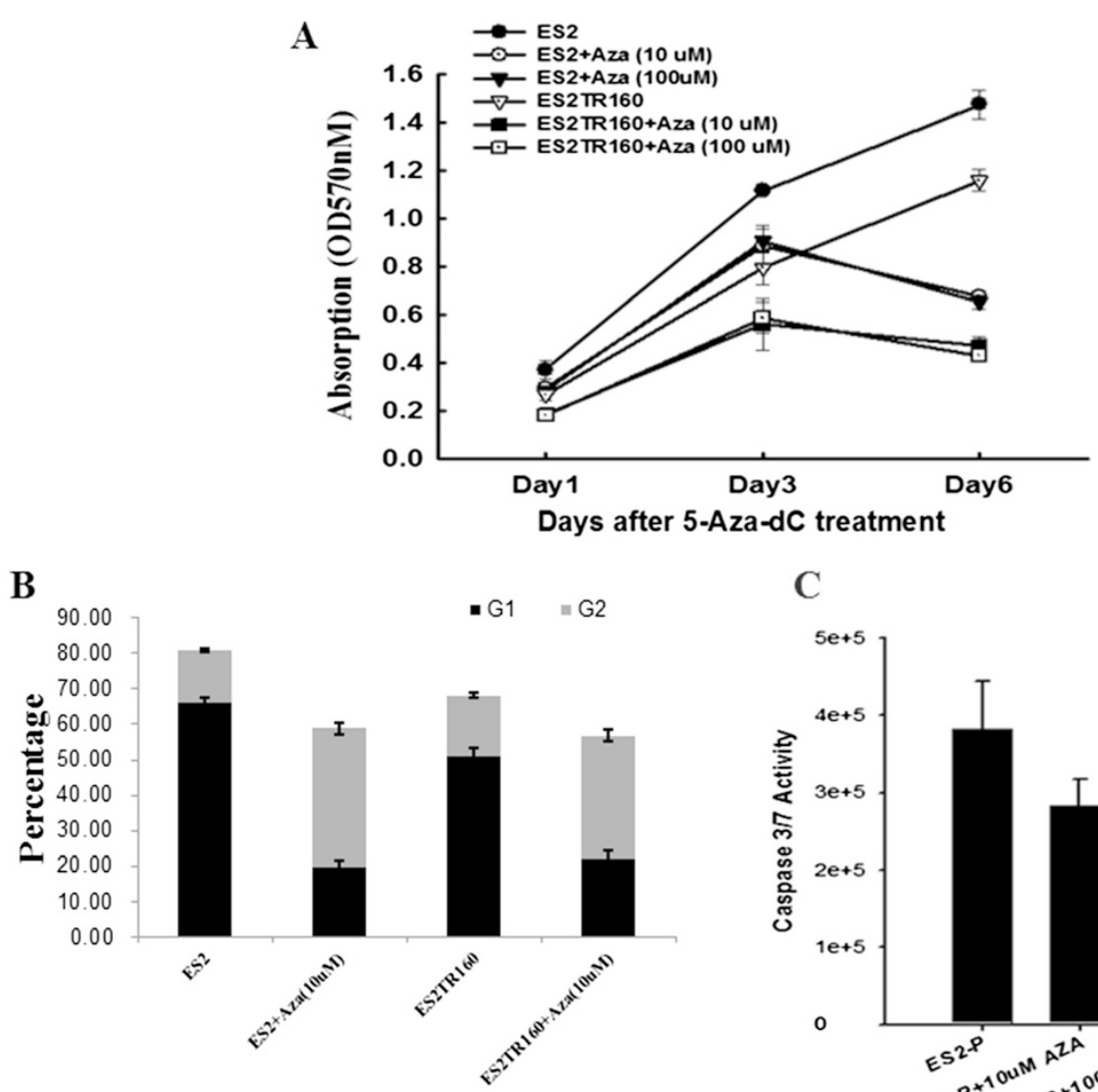

$\mathrm{C}$

Fig. 6 a The effect of $10 \mu \mathrm{M}$ or $100 \mu \mathrm{M}$ of 5-aza-2-dC on in vitro cell growth of ES2 and ES2TR160 cells by MTT assay. $\mathbf{b}$ The percentages of G1 and G2 phases in both ES2 and ES2TR160 cells with 5-aza-2-dC treatment for 3 days. c The caspase-3/7 activity in both ES2 and ES2TR160 cells with or without 5-aza-2-dC treatment

\begin{tabular}{|c|c|}
\hline $\mathbf{A}$ & ES2 ES2TR160 \\
\hline & $10 \mu \mathrm{M} 5-\mathrm{AZA}(-)(+)(-)(+)$ \\
\hline 123 & HIN1 $\square-\cdots$ \\
\hline HIN1 --- & AKT $\square$ \\
\hline AKT $\square$ & p-AKT $\left(\right.$ Thr $\left.^{308}\right) \square$ \\
\hline p-AKT $\left(T_{h r}{ }^{308}\right) \square$ & p-AKT $\left(\operatorname{Ser}^{473}\right) \square=$ \\
\hline p-AKT $\left(\operatorname{Ser}^{473}\right) \square-$ & mTOR जमEब \\
\hline mTOR $=-\square$ & \\
\hline $\mathrm{p}-\mathrm{mTOR}$ & p-mTOR \\
\hline GAPDH & GAPDH \\
\hline \multicolumn{2}{|c|}{$\begin{array}{l}\text { Fig. } 7 \text { a Changes in HIN-1, p-AKT (Thr308), p-AKT (Ser473), and } \\
\text { p-mTOR protein expressions in ES2 mock, ES2TR160 mock, and } \\
\text { ES2TR160HIN-1 transfectants by Western blotting. 1: ES2 mock, 2: } \\
\text { ES2TR160 mock, 3: ES2TR160HIN-1 transfectant. b HIN-1, p-AKT } \\
\text { (Thr308), p-AKT (Ser473), and p-mTOR protein expressions in ES2 and } \\
\text { ES2TR160 cells treated with or without 5-aza-2-dC by Western blotting }\end{array}$} \\
\hline
\end{tabular}

2-dC-treated paclitaxel-resistant ES2TR160 cells (Fig. 7b). These results demonstrated that 5-aza-2-dC may increase the expression of HIN-1 by decreasing the AKT-mTOR expression in paclitaxel-resistant OCCC tumor cells.

\section{Discussion}

Aberrations in DNA methylation are involved in tumor progression and the acquisition of drug resistance. To investigate whether paclitaxel selects preferentially for DNA methylation in chemo-resistant OCCC cell lines, we used MS-MLPA to detect 40 tumor suppressing genes (TSGs) in ES2 and TOV21G parental and resistant cells. We found changes in methylation of the HIN-1 gene in ES2TR160 and TV21GTR200 paclitaxel-resistant cells, and this may be involved in the mechanism of paclitaxel resistance (data not shown). Significantly more paclitaxelresistant OCCC cells had a low expression of the HIN-1 protein compared to the paclitaxel-sensitive OCCC cells $(93.8 \%$ vs. $62.8 \%, p=0.03)$, suggesting that downregulation of the expression of HIN-1 is strongly correlated with paclitaxel-resistant OCCC tumors. Over-expression of 
the HIN-1 gene effectively decreased the tumor growth of paclitaxel-resistant ES2TR160 tumor cells, which is consistent with promoter methylation of $\mathrm{HIN}-1$ and the poor outcomes of patients with OCCC [16]. 5-Aza-2-dC inhibited tumor growth by demethylating aberrantly methylated TSGs and maintaining function, presumably through restoration of HIN-1 expression with a decrease in AKT-mTOR expression. Furthermore, 5-aza-2-dC inhibited growth of ES2 and ES2TR160 cells mainly by inhibiting the G2M phase, but without increasing apoptosis and autophagy (Fig. 6). These results support the concept that 5-aza-2-dC can inhibit tumor growth of OCCC partly through affecting the HIN-1-related AKT-mTOR signaling pathway, and this may be a promising therapy for the management of primary or recurrent OCCC. Clinical trials are warranted to test this hypothesis.

OCCC is a chemo-resistant tumor. Experimental evidence has suggested different mechanisms by which tumor cells can develop resistance to taxanes, including excluding taxane from cells by ATP-binding cassette transporters, the expression of certain tubulin isoforms and microtubule associated proteins, tubulin gene mutations, alterations in survival or mitotic check point signaling, and methylationassociated Has-miR-9 deregulation [17, 18]. ES2TR160 and TOV21GTR200 cancer cell lines did not acquire paclitaxel resistance via somatic mutations in tubulin genes, as those nicely reviewed in previous report [18]. In the present study, however, G2-M phase arrest was absent in the paclitaxel-resistant OCCC cells even though these cells were under paclitaxel treatment. Our results support that the mechanism of paclitaxel resistance in OCCC may involve the drug transporter gene, cancer stem cell characteristics, hypoxic tumor microenvironment, and epithelial-mesenchymal transition.

Methylation of HIN-1 is involved in the chemoresistance of OCCC. Importantly, the reversal of HIN-1 epigenetic silencing by demethylation or over-expression of the HIN-1 gene was demonstrated to resensitize tumor cells to paclitaxel treatment in vitro and in vivo. We also observed that CpG sites at probe 12956 of the HIN-1 gene were hypomethylated in ES2 and TOV21G cells, whereas they became hypermethylated following step-wise exposure to paclitaxel in ES2TR160 and TOV21GTR200 cells as confirmed by methylation-specific PCR, suggesting that hypermethylation occurs in acquired paclitaxel chemo-resistance (data not shown).

We previously showed that the ectopic expression of the HIN-1 gene increases paclitaxel sensitivity, partly through the Akt pathway [16]. The SCGB3A1 gene, also called HIN-1 (high in normal-1), encodes a small secreted protein, secretoglobin $3 \mathrm{~A} 1$ which is a member of the secretoglobin family [19]. Recent reports have shown that HIN-1 expression is down-regulated in the majority of lung, breast, prostate, pancreatic, colorectal, testicular and nasopharyngeal cancers, and that this down-regulation is associated with hypermethylation of the HIN-1 promoter [20-24]. Thus, silencing of HIN-1 expression by methylation is an early and frequent event in multiple human types of cancer, and is functionally relevant to tumorigenesis [22]. These findings together with in vitro data on growth inhibition and AKT activation in breast cancer suggest that HIN-1 may be a candidate tumor suppressor gene [24].

Clinical studies have shown that a low dose of decitabine can alter the DNA methylation of genes and cancer pathways, thereby restoring sensitivity to carboplatin in heavily pretreated ovarian cancer patients who progressed or recurred within 6 months after platinum-based chemotherapy, resulting in a high response rate and prolonged progression free survival [12]. The selective epigenetic disruption of distinct biological pathways has been observed during the development of platinum resistance in patients with ovarian cancer. Hypermethylation-mediated repression of cell adhesion and tight junction pathways, and hypomethylation-mediated activation of the cell growthpromoting pathways PI3K/Akt and TGF-beta, and cell cycle progression may contribute to the onset of chemoresistance in ovarian cancer cells [15].

The PI3K/Akt pathway has been shown to contribute to cisplatin resistance by promoting cell proliferation and increasing drug metabolism and resistance to apoptosis $[25,26]$. Paclitaxel activates AKT and mTORC1 signaling which act as resistant factors and protect cancer cells from death/apoptosis [27, 28]. The mammalian target of rapamycin (mTOR) has been identified to be a downstream target of the PI3K/Akt pathway, and it has emerged as a critical effector in cell signaling pathways commonly deregulated in human cancers. mTOR has been reported to be phosphorylated and activated in endometriosis and OCCC specimens [29]. This leads to phosphorylation of downstream targets, p70S6K and 4E-BP1, and the subsequent enhanced translation of mRNA that is critical for cell cycle progression and proliferation. Recently, a therapeutic strategy targeting the mTOR-HIF- $1 \alpha$-VEGF pathway in OCCC has been proposed based on the finding that p-mTOR expression is more prominent in OCCC than ovarian serous carcinoma [30]. After treatment with an analogue of rapamycin (everolimus), the expressions of p-mTOR, HIF- $1 \alpha$ and VEGF were shown to be sharply decreased [30].

In this study, 5-aza-2-dC not only decreased phosphoAKT at Thr308 and Ser473 and phospho-mTOR, but also restored HIN-1 expression in paclitaxel-resistant cells in vitro. In addition, treatment with $10 \mu \mathrm{M}$ 5-aza-2$\mathrm{dC}$ also inhibited the growth of both ES2 and ES2TR160 tumor cells (Fig. 6a). Furthermore, 5-aza-2-dC treatment significantly reduced the percentage of the G1 phase in both ES2 and ES2TR160 cells after 3 days of treatment. These results support that 5 -aza-2-dC can overcome 
paclitaxel drug resistance and inhibit tumor growth of paclitaxel-resistant OCCC partly through affecting the HIN-1-AKT-mTOR signaling pathway.

Taken together, a demethylating agent can restore the HIN-1 expression in paclitaxel-resistant OCCC cells through the HIN-1-AKT-mTOR signaling pathway and then inhibit in vivo tumor growth. Restoration of HIN-1 by a demethylating agent may be a potential strategy for the treatment of paclitaxel-resistant OCCC.

\section{Conclusion}

This is the first study to show that a demethylating agent could inhibit tumor growth of OCCC in vivo and in vitro. Treatment with 5-aza-2-dC remarkably inhibited ES2derived tumor growth by $90-100 \%$ compared to the controls, in both ES2 (paclitaxel-sensitive) and ES2TR160 (paclitaxel-resistant) cells treated with 5-aza-2-dC for 3 days prior to subcutaneous inoculation in mice. We concluded that 5-aza-2-dC has a remarkable anti-tumor effect as a single agent in OCCC.

\section{Abbreviations}

OCCC: Ovarian clear cell carcinoma; 5-aza-2-dC: 5-aza-2-deoxycytidine.

\section{Competing interests}

The authors declare that they have no competing interests.

\section{Authors' contributions}

Conceived and designed the experiments: CMH, SFC, WFC. Perform the experiments: $\mathrm{CJH}, \mathrm{SHH}$. Analyzed the data: $\mathrm{CMH}$, SFC. Wrote the paper: $\mathrm{CMH}$, WFC. All authors read, and approved the final manuscript.

\section{Acknowledgements}

This work was supported by research funds from the National Science Council and Cathay General Hospital, Taipei, Taiwan (NSC101-101-2314-B281-005-MY3, 103-CGH-TMU-07, 102CGH-TMU-06, CGH-MR10226).

\section{Author details \\ ${ }^{1}$ Gynecologic Cancer Center, Department of Obstetrics and Gynecology, Cathay General Hospital, Taipei, Taiwan. ${ }^{2}$ School of Medicine, Fu Jen Catholic University, Hsinchuang, New Taipei City, Taiwan. ${ }^{3}$ School of Medicine, Taipei Medical University, Taipei, Taiwan. ${ }^{4}$ Department of Medical Research, Cathay General Hospital, Sijhih, New Taipei, Taiwan. ${ }^{5}$ Department of Pathology, Cathay General Hospital, Taipei, Taiwan. ${ }^{6}$ Graduate Institute of Medical Sciences, School of Medicine, Taipei Medical University, Taipei, Taiwan. ${ }^{7}$ Department of Obstetrics and Gynecology, National Taiwan, University Hospital, Taipei, Taiwan. ${ }^{8}$ Graduate Institute of Oncology, National Taiwan, University Hospital, Taipei, Taiwan. ${ }^{9}$ Graduate Institute of Clinical Medicine, College of Medicine, National Taiwan, University, Taipei, Taiwan. \\ ${ }^{10}$ Department of Biochemistry, National Defense Medical Center, Taipei, Taiwan.}

Received: 9 June 2015 Accepted: 9 October 2015

Published online: 24 October 2015

\section{References}

1. Jemal A, Siegel R, Ward E, Hao Y, Xu J, Murray T, et al. Cancer statistics, 2008. CA Cancer J Clin. 2008;58:71-96.

2. Ozols RF, Bundy BN, Greer BE, Fowler JM, Clarke-Pearson D, Burger RA, et al. Phase III trial of carboplatin and paclitaxel compared with cisplatin and paclitaxel in patients with optimally resected stage III ovarian cancer: a gynecologic oncology group study. J Clin Oncol. 2003;21:3194-200.

3. Köbel M, Kalloger SE, Huntsman DG, Santos JL, Swenerton KD, Seidman JD, et al. Differences in tumor type in low-stage versus high-stage ovarian carcinomas. Int J Gynecol Pathol. 2010;29:203-11.
4. Chiang YC, Chen CA, Chiang CJ, Hsu TH, Lin MC, You SL, et al. Trends in incidence and survival outcome of epithelial ovarian cancer: 30-year national population-based registry in Taiwan. J Gynecol Oncol. 2013;24:342-51.

5. Ho CM, Huang YJ, Chen TC, Huang SH, Liu FS, Chang Chien CC, et al. Pure-type clear cell carcinoma of the ovary as a distinct histological type and improved survival in patients treated with paclitaxel platinum-based chemotherapy in pure-type advanced disease. Gynecol Oncol. 2004;94:197.203.

6. Itamochi H, Kigawa J, Terakawa N. Mechanisms of chemoresistance and poor prognosis in ovarian clear cell carcinoma. Cancer Sci. 2008;99:653-8.

7. Bookman MA, Greer BE, Ozols RF. Optimal therapy of advanced ovarian cancer: carboplatin and paclitaxel vs. cisplatin and paclitaxel (GOG158) and an update on GOG0182-ICON5. Int J Gynecol Cancer. 2003;13:735-40.

8. Sugiyama T, Kamura T, Kigawa J, Terakawa N, Kikuchi Y, Kita T, et al. Clinical characteristics of clear cell carcinoma of the ovary: a distinct histological type with poor prognosis and resistance to platinum-based chemotherapy. Cancer. 2000;88:2584-549.

9. Ho CM, Chien TY, Shih BY, Huang SH. Evaluation of complete surgical staging with pelvic and para-aortic lymphadenectomy and paclitaxel plus carboplatin chemotherapy for improvement of survival in stage I ovarian clear cell carcinoma. Gynecol Oncol. 2003;88:394-9.

10. Crotzer DR, Sun CC, Coleman RL, Wolf JK, Levenback CF, Gershenson DM. Lack of effective systemic therapy for recurrent clear cell carcinoma of the ovary. Gynecol Oncol. 2007;105:404-8.

11. Zeller C, Dai W, Steele NL, Siddiq A, Walley AJ, Wilhelm-Benartzi CS, et al. Candidate DNA methylation drivers of acquired cisplatin resistance in ovarian cancer identified by methylome and expression profiling. Oncogene. 2012;31:4567-76.

12. Plumb JA, Strathdee G, Sludden J, Kaye SB, Brown R. Reversal of drug resistance in human tumor xenografts by 20-deoxy-5-azacytidine azacytidine induced demethylation of the hMLH1 gene promoter. Cancer Res. 2000;60:6039-44.

13. Balch C, Yan P, Craft T, Young S, Skalnik DG, Huang TH, et al. Antimitogenic and chemosensitizing effects of the methylation inhibitor zebularine in ovarian cancer. Mol Cancer Ther. 2005;4:1505-14.

14. Li M, Balch C, Li Y, Hu W, Shen DY, Kavanagh JJ, et al. Azacitidine enhances sensitivity of platinum-resistant ovarian cancer cells to carboplatin through induction of apoptosis. Am J Obstet Gynecol. 2009;200:177e1-9.

15. Ho CM, Huang CJ, Huang CY, Wu YY, Chang SF, Cheng WF. Promoter Methylation status of $\mathrm{HIN-1}$ associated with outcomes of ovarian clear cell adenocarcinoma. Mol Cancer. 2012;11:53.

16. Chien AJ, Moasser MM. Cellular mechanisms of resistance to anthracyclines and taxanes in cancer: intrinsic and acquired. Semin Oncol. 2008;35:S1-S14.

17. Li X, Pan Q, Wan X, Mao Y, Lu W, Xie X, et al. Methylation-associated Has-miR-9 deregulation in paclitaxel- resistant epithelial ovarian carcinoma. BMC Cancer. 2015;15:509.

18. Orr GA, Verdier Pinard P, McDaid H, Horwitz SB. Mechanisms of Taxol resistance related to microtubules. Oncogene. 2003;22:7280-95.

19. Singh G, Katyal SL. Clara cell proteins. Ann NY Acad Sci. 2000;923:43-58.

20. Wong TS, Kwong DL, Sham JS, Tsao SW, Wei WI, Kwong YL, et al. Promoter hypermethylation of high-in-normal 1 gene in primary nasopharyngeal carcinoma. Clin Cancer Res. 2003;9:3042-6.

21. Yu Y, Yin D, Hoque MO, Cao B, Jia Y, Yang Y, et al. AKT signaling pathway activated by HIN-1 methylation in non-small cell lung cancer. Tumour Biol. 2012;33:307-14.

22. Marchetti A, Barassi F, Martella C, Chella A, Salvatore S, Castrataro A, et al. Down regulation of high in normal-1 (HIN-1) is a frequent event in stage I non-small cell lung cancer and correlates with poor clinical outcome. Clin Cancer Res. 2004;10:1338-43.

23. Shigematsu H, Suzuki M, Takahashi T, Miyajima K, Toyooka S, Shivapurkar $N_{\text {, }}$ et al. Aberrant methylation of $\mathrm{HIN-1}$ (high in normal-1) is a frequent event in many human malignancies. Int J Cancer. 2005;113:600-4.

24. Krop I, Player A, Tablante A, Taylor-Parker M, Lahti-Domenici J, Fukuoka J, et al. Frequent $\mathrm{HIN}-1$ promoter methylation and lack of expression in multiple human tumor types. Mol Cancer Res. 2004:2:489-94.

25. Hennessy BT, Smith DL, Ram PT, Lu Y, Mills G. Exploiting the PI3K/AKT pathway for cancer drug discovery. Nat Rev Drug Discov. 2005;4:988-1004.

26. Zhang HY, Zhang PN, Sun H. Aberration of the PI3K/AKT/mTOR signaling in epithelial ovarian cancer and its implication in cisplatin-based chemotherapy. Eur J Obstet Gynecol Reprod Biol. 2009;146:81-6.

27. Bava SV, Puliappadamba VT, Deepti A, Nair A, Karunagaran D, Anto RJ. Sensitization of taxol-induced apoptosis by curcumin involves down-regulation 
of nuclear factor-kappaB and the serine/threonine kinase Akt and is independent of tubulin polymerization. J Biol Chem. 2005;280:6301-8.

28. Lin HL, Lui WY, Liu TY, Chi CW. Reversal of Taxol resistance in hepatoma by cyclosporin A: involvement of the Pl-3 kinase-AKT 1 pathway. Br J Cancer. 2003;88:973-80.

29. Yagyu T, Tsuji Y, Haruta S, Kitanaka T, Yamada Y, Kawaguchi R, et al. Activation of mammalian target of rapamycin in postmenopausal ovarian endometriosis. Int J Gynecol Cancer. 2006;16:1545-51.

30. Miyazawa M, Yasuda M, Fujita M, Kajiwara H, Hirabayashi K, Takekoshi S, et al. Therapeutic strategy targeting the mTOR-HIF-1alpha-VEGF pathway in ovarian clear cell adenocarcinoma. Pathol Int. 2009;59:19-27.

\section{Submit your next manuscript to BioMed Central} and take full advantage of:

- Convenient online submission

- Thorough peer review

- No space constraints or color figure charges

- Immediate publication on acceptance

- Inclusion in PubMed, CAS, Scopus and Google Scholar

- Research which is freely available for redistribution 